\title{
Embed Longest Rings onto Star Graphs with Vertex Faults
}

\author{
Sun-Yuan Hsieh, Gen-Huey Chen \\ Department of Computer Science and Information Engineering \\ National Taiwan University, Taipei, Taiwan, ROC \\ and \\ Chin-Wen Ho \\ Department of Computer Science and Information Engineering \\ National Central University, Taipei, Taiwan, ROC
}

\begin{abstract}
The star graph has been recognized as an attractive alternative to the hypercube. Let $F_{e}$ and $F_{v}$ be the sets of vertex faults and edge faults, respectively. Previously, Tseng et al. showed that an n-dimensional star graph can embed a ring of length $n !$ if $\left|F_{e}\right| \leq n-3\left(\left|F_{v}\right|=0\right)$, and a ring of length at least $n !-4\left|F_{v}\right|$ if $\left|F_{v}\right| \leq n-3 \quad\left(\left|F_{e}\right|=0\right)$. Since an $n$-dimensional star graph is regular of degree $n-1$ and is bipartite with two partite sets of equal size, our result achieves optimality in the worst case.
\end{abstract}

\section{Introduction}

One crucial step on designing a large-scale multiprocessor system is to determine the topology of the interconnection network (network for short). In recent decades, a lot of networks have been proposed. The interested readers may refer to $[4,17]$ for extensive references. Among them, the star graph [1], which belongs to the class of Cayley graphs [2], has been recognized as an attractive alternative to the hypercube. It possesses many nice topological properties such as recursiveness, symmetry, maximal fault tolerance, sublogarithmic degree and diameter, and strong resilience, which are all desirable when we are building interconnection topologies for parallel and distributed systems. Besides, the star graph can embed rings [25], meshes [27], trees [3], and hypercubes [23]. Efficient communication algorithms for shortest-path routing [1], multiple-path routing [12], multicasting [16], broadcasting [31], gossiping [5], and scattering [16] have been proposed. Efficient algorithms for sorting [22], merging [25], selection [24], prefix sums [25], ranking [30], Fourier transform [15], and computational geometry [26] have also been designed.

Since processor and/or link faults may happen when a network is put in use, it is practically meaningful to consider faulty networks. The problems of diameter [28], routing [14, 34], multicasting [21], broadcasting [33], gossiping [13], embedding [11, 29, 35], and fault-tolerant graphs [6-9, 19] have been solved on a variety of faulty networks. This paper is concerned with the problem of embedding a ring onto a faulty star graph. Throughout this paper, we use network and graph, processor and vertex, and link and edge, interchangeably.

Previously, embedding rings onto faulty star graphs has been studied in [20, 32]. In [20], Latifi and Bagherzadeh showed that an $n$-dimensional star graph with vertex faults can embed a ring of length $n !-m$ !, where all vertex faults belong to an $m$-dimensional star graph and $m \leq n$ is minimal. Letting $F_{v}$ and $F_{e}$ denote the sets of vertex faults and edge faults, respectively, Tseng et al. [32] showed that an $n$-dimensional star graph can embed a ring of length $n$ ! if $\left|F_{e}\right| \leq n-3\left(\left|F_{v}\right|=0\right)$, and a ring of length at least $n !-4\left|F_{v}\right|$ if $\left|F_{v}\right| \leq n-3 \quad\left(\left|F_{e}\right|=0\right)$. Since the $n$-dimensional star graph is regular of $n-1$ and contains $n$ ! vertices, the former is optimal in the worst case. However, the latter is not optimal.

In this paper, we improve Tseng et al.'s latter result by lengthening the embedded ring from $n !-4\left|F_{v}\right|$ to $n !-2\left|F_{v}\right|$. We note that the star graph is bipartite and has two partite sets of equal size [18]. When $\left|F_{v}\right|$ vertex faults fall into the same partite set, an embedded ring has length not more than $n !-2\left|F_{v}\right|$. Hence, our result achieves optimality in the worst case. 


\section{Preliminaries}

In this section the structure of the star graph is formally described. Some necessary notations and basic operations are also introduced. We use $S_{n}$ to denote an $n$-dimensional star graph.

Definition 1. The vertex set of $S_{n}$ is denoted by $\left\{a_{1} a_{2} \ldots a_{n} \mid a_{1} a_{2} \ldots a_{n}\right.$ is a permutation of $\left.1,2, \ldots, n\right\}$. Vertex adjacency is defined as follows: $a_{1} a_{2} \ldots a_{n}$ is adjacent to $a_{i} a_{2} \ldots a_{i-1} a_{1} a_{i+1} \ldots a_{n}$ for all $2 \leq i \leq n$.

The vertices of $S_{n}$ are $n$ ! permutations of $1,2, \ldots, n$, and there is an edge between two vertices of $S_{n}$ if and only if they can be obtained from each other by swapping the leftmost number with one of the other $n$-1 numbers. We refer to the position of $a_{i}$ in $a_{1} a_{2} \ldots a_{n}$ as the ith dimension, and the edge between $a_{1} a_{2} \ldots a_{n}$ and $a_{i} a_{2} \ldots a_{i-1} a_{1} a_{i+1} \ldots a_{n}$ as the ith-dimensional edge. Clearly $S_{n}$ is regular of degree $n$ 1. Since $S_{1}$ is a vertex, $S_{2}$ is an edge, and $S_{3}$ forms a cycle of length 6 , we consider $S_{n}$ with $n \geq 4$ throughout this paper.

There are embedded $S_{r}$ 's contained in $S_{n}$, where $1 \leq r \leq n$. An embedded $S_{r}$ can be conveniently represented by $\left\langle s_{1} s_{2} \ldots s_{n}\right\rangle_{r}$, where $s_{1}=*, s_{i} \in\{*, 1,2, \ldots, n\}$ for all $2 \leq i \leq n$, and exactly $r$ of $s_{1}, s_{2}, \ldots, s_{n}$ are $*(*$ denotes a "don't care" symbol). For example, $\langle * * * 3\rangle_{3}$, which represents an embedded $S_{3}$ in $S_{4}$, contains six nodes $1243,1423,2143$, 2413,4123 , and 4213 . In terms of graph, $\langle * * * 3\rangle_{3}$ is a subgraph of $S_{4}$ induced by $\{1243,1423,2143,2413,4123$, $4213\}$. When $r=n,\left\langle s_{1} s_{2} \ldots s_{n}\right\rangle_{n}$ represents $S_{n}$. Two basic operations on $S_{n}$ are defined as follows.

Definition 2. An i-partition on $\left\langle s_{1} s_{2} \ldots s_{n}\right\rangle_{r}$ partitions $\left\langle s_{1} s_{2} \ldots s_{n}\right\rangle_{r}$ into $r$ embedded $S_{r-1}$ 's, denoted by $\left\langle s_{1} s_{2} \ldots s_{i-}\right.$ ${ }_{1} q s_{i+1} \ldots s_{n}>_{r-1}$, where $2 \leq i \leq n, s_{i}=*$, and $q \in\{1,2, \ldots, n\}-\left\{s_{1}\right.$, $\left.s_{2}, \ldots, s_{n}\right\}$.

For example, executing a 3-partition on $\langle * * * 15\rangle_{3}$ produces three embedded $S_{2}$ 's, i.e., $\langle * * 215\rangle_{2},\langle * * 315\rangle_{2}$, and $\langle * * 415\rangle_{2}$.

Definition 3. An $\left(i_{1}, i_{2}, \ldots, i_{m}\right)$-partition on $\left\langle s_{1} s_{2} \ldots s_{n}\right\rangle_{r}$ performs an $i_{1}$-partition, an $i_{2}$-partition, ..., an $i_{m}$-partition, sequentially, on $\left\langle s_{1} s_{2} \ldots s_{n}\right\rangle_{r}$, where $i_{1} i_{2} \ldots i_{m}$ is a permutation of $m$ elements from $\{2,3, \ldots, n\}$ and $s_{i_{1}}=s_{i_{2}}=\cdots=s_{i_{m}}=*$. After executing an $\left(i_{1}, i_{2}, \ldots, i_{m}\right)$-partition, $\left\langle s_{1} s_{2} \ldots s_{n}\right\rangle_{r}$ is partitioned into $r(r-1) \cdots(r-m+1)$ embedded $S_{r-m}$ 's.

For example, when a $(3,2)$-partition is applied to $\langle * * * 15\rangle_{3}$, a 3 -partition is first executed on $\langle * * * 15\rangle_{3}$ to produce three embedded $S_{2}$ 's, i.e., $\langle * * 215\rangle_{2},\langle * * 315\rangle_{2}$, and $\langle * * 415\rangle_{2}$. Then, a 2-partition is executed on each of these three $S_{2}$ 's to produce six embedded $S_{1}$ 's, i.e., $\langle * 3215\rangle_{1},\langle * 4215\rangle_{1},\langle * 2315\rangle_{1},\langle * 4315\rangle_{1},\langle * 2415\rangle_{1}$, and $\langle * 3415\rangle_{1}$.

Two embedded $S_{r}$ 's $\left\langle s_{1} s_{2} \ldots s_{n}\right\rangle_{r}$ and $\left\langle t_{1} t_{2} \ldots t_{n}\right\rangle_{r}$ are said to be adjacent if $s_{j} \neq *, t_{j} \neq *$, and $s_{j} \neq t_{j}$ for some $2 \leq j \leq n$, and $s_{i}=t_{i}$ for all $1 \leq i \leq n$ and $i \neq j$. Moreover, the position $j$ is denoted by $\operatorname{dif}\left(\left\langle s_{1} s_{2} \ldots s_{n}\right\rangle_{r},\left\langle t_{1} t_{2} \ldots t_{n}\right\rangle_{r}\right)$. For example, $\langle * * 23\rangle_{2}$ is adjacent to $\langle * * 13\rangle_{2}$, and $\operatorname{dif}\left(\langle * * 23\rangle_{2}\right.$, $\left.<* * 13\rangle_{2}\right)=3$.

In the rest of this paper, an embedded $S_{r}$ is named an $r$-vertex when it is regarded as a supervertex. Imaginarily, there is a superedge, named an r-edge, between two adjacent $r$-vertices. Really, an $r$-edge in $S_{n}$ comprises $(r-1)$ ! edges. A 1 -vertex in $S_{n}$ is a vertex of $S_{n}$, and a 1-edge in $S_{n}$ is an edge of $S_{n}$.

Definition 4. Suppose $A_{0}, A_{1}, \ldots, A_{n(n-1)(n-2) \cdots(r+1)-1}$ are $n(n-1)(n-2) \cdots(r+1) r$-vertices that result from executing an $\left(i_{1}, i_{2}, \ldots, i_{n-r}\right)$-partition on $S_{n}$, where $1 \leq r \leq n-1$. They form an $r$-ring, denoted by $R_{r}=\left[A_{0}, A_{1}, \ldots, A_{n(n-1)(n-2) \cdots(r+1)-1}\right]$, if $A_{i}$ is adjacent to $A_{(i-1) \bmod n(n-1)(n-2) \cdots(r+1)}$ and $A_{(i+1) \bmod n(n-1)(n-2) \cdots(r+1)}$ for all $0 \leq i \leq n(n-1)(n-2) \cdots(r+1)-1$.

Definition 5. An $i$-partition on an $R_{r}=\left[A_{0}, A_{1}, \ldots, A_{n(n-}\right.$

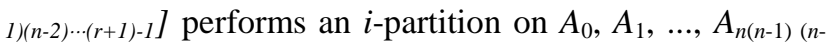
2) $\cdots(r+1)-1$, where $2 \leq i \leq n$ and $r \geq 2$. An $i$-partition on an $R_{r}$ is abbreviated to a partition on an $R_{r}$ if the position $i$ is "don't care".

After executing an $i$-partition on an $R_{r}=\left[A_{0}, A_{1}, \ldots, A_{n(n-}\right.$

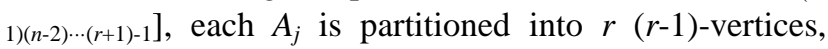
where $0 \leq j \leq n(n-1)(n-2) \cdots(r+1)-1$. Since every two of the $r$ $(r-1)$-vertices are adjacent, each $A_{j}$ can be viewed as a complete graph of $r(r$-1)-vertices. Throughout this paper, we use $K_{r}^{r-1}$ to denote the complete graph.

Suppose $A_{k}=\left\langle s_{1} \ldots s_{i-1} s_{i} s_{i+1} \ldots s_{j-1} x s_{j+1} \ldots s_{n}\right\rangle_{r}$ and $A_{(k+1) \text { mod }}$ $n(n-1)(n-2) \cdots(r+1)=\left\langle s_{1} \ldots s_{i-1} s_{i} s_{i+1} \ldots s_{j-1} y s_{j+1} \ldots s_{n}\right\rangle_{r} \quad$ are two neighboring $r$-vertices in an $R_{r}$, where $r \geq 2, x \neq y$, and $s_{i}=*$. After an $i$-partition, they each are partitioned into $r(r-1)$ vertices. Among them, $r$-1 from $A_{k}$ are adjacent to $r$-1 from $A_{(k+1) \bmod n(n-1)(n-2) \cdots(r+1)}$. The other two that are not adjacent are $\left\langle s_{1} \ldots s_{i-1} y s_{i+1} \ldots s_{j-1} x s_{j+1} \ldots s_{n}\right\rangle_{r-1}$ and $\left\langle s_{1} \ldots s_{i-1} x s_{i+1} \ldots s_{j-1} y s_{j+1} \ldots\right.$ $s_{n}>_{r-1}$.

Suppose an $R_{k}=\left[A_{0}, A_{1}, \ldots, A_{n(n-1)(n-2) \cdots(k+1)-1}\right]$ is obtained in $S_{n}$, where $k \geq 4$. After executing a partition on the $R_{k}$, each $A_{i}$ forms a $K_{k}^{k-1}$, where $0 \leq i \leq n(n-1) \cdots(k+1)-1$. There exist two $(k-1)$-vertices, say $X_{i}$ and $Y_{i}$, in each $A_{i}$ so that $X_{i}$ and $Y_{i}$ are adjacent to $Y_{(i-1) \bmod n(n-1) \cdots(k+1)}$ and $X_{(i+1) \bmod n(n-1) \cdots(k+1)}$, respectively. We refer to $X_{i}$ and $Y_{i}$ as the entry $(k-1)$-vertex and exit $(k-1)$-vertex of $A_{i}$, respectively, in subsequent discussion. 


\section{Embed a longest ring onto $S_{n}$ with vertex faults}

In this section we assume that $S_{n}$ has $\left|F_{v}\right| \leq n-3$ vertex faults. We say that an $i$-vertex is healthy if it has no vertex fault, and faulty otherwise, where $1 \leq i \leq n$. Similarly, a ring (or a path) is healthy if it has no vertex fault. In this section we show that $S_{n}$ with $n \geq 4$ can embed a healthy ring of length $n !-2\left|F_{v}\right|$. Since $S_{n}$ is bipartite with two partite sets of equal size, the length $n !-2\left|F_{v}\right|$ is maximum in the worst case. Moreover, $\left|F_{v}\right|=n-3$ is also maximum in the worst case in order to embed a healthy ring of maximum length, because $S_{n}$ is regular of $n-1$. Our method is first to determine an $R_{4}$ in $S_{n}$ that owns the following three properties:

(P1) each 4-vertex of the $R_{4}$ has at most one vertex fault;

(P2) for every three consecutive 4-vertices $U=\left\langle u_{1} u_{2} \ldots u_{n}\right\rangle_{4}, \quad V=\left\langle v_{1} v_{2} \ldots v_{n}\right\rangle_{4}, \quad$ and $W=\left\langle w_{1} w_{2} \ldots w_{n}>_{4}\right.$ in the $R_{4}, u_{\operatorname{dif}(U, V)} \neq w_{\operatorname{dif}(V, W)}$ holds;

(P3) both every two consecutive 4-vertices in the $R_{4}$ are not faulty.

Then, a healthy ring of length $n !-2\left|F_{v}\right|$ can be generated from the $R_{4}$.

Lemma 1. Suppose $U=\left\langle u_{1} u_{2} \ldots u_{n}\right\rangle_{r}, V=\left\langle v_{1} v_{2} \ldots v_{n}\right\rangle_{r}$, and $W=\left\langle w_{1} w_{2} \ldots w_{n}\right\rangle_{r}$ are arbitrary three consecutive $r$ vertices in an $R_{r}$, where $r \geq 2$. Let $p=\operatorname{dif}(U, V)$ and $q=\operatorname{dif}(V$, $W)$. If $u_{p} \neq w_{q}$, then after executing a partition on the $R_{r}$, each $(r-1)$-vertex of $V$ is connected to $U$ or $W$.

Proof. Without loss of generality, we assume that a $j$ partition is executed on the $R_{r}$, where $2 \leq j \leq n$. Hence, $u_{j}=v_{j}=w_{j}=*$. Since $p=\operatorname{dif}(U, V) \neq 1$ and $q=\operatorname{dif}(V, W) \neq 1$, we have $u_{p} \neq v_{p}, v_{q} \neq w_{q}, u_{i}=v_{i}$ for all $1 \leq i \leq n$ and $i \neq p$, and $v_{i}=w_{i}$ for all $1 \leq i \leq n$ and $i \neq q$. Suppose conversely that $u_{p} \neq w_{q}$ and there exists an $(r-1)$-vertex, say $V_{1}=\left\langle v_{1} v_{2} \ldots v_{j-1} z v_{j+1} \ldots v_{n}\right\rangle_{r-1}$, of $V$, which is connected to neither of $U$ and $W$. Thus, $z=u_{p}$, for otherwise $V_{1}$ is adjacent to some $(r-1)$-vertex of $U$. Similarly, $z=w_{q}$. Hence, $u_{p}=z=w_{q}$, which contradicts our assumption.

Q.E.D.

The following lemma was shown in [32].

Lemma 2.[32] Suppose $\left|F_{v}\right| \leq n-3$. There exists a sequence $a_{1}, a_{2}, \ldots, a_{n-4}$ of positions so that after executing an $\left(a_{1}, a_{2}, \ldots, a_{n-4}\right)$-partition on $S_{n}$, each resulting 4-vertex contains at most one vertex fault.

The positions $a_{1}, a_{2}, \ldots, a_{n-4}$ in Lemma 2 can be easily determined as follows. We let $a_{1}$ be a position where at least two vertices in $F_{v}$ differ. For example, if $F_{v}=\{123456$,
123654 \}, $a_{1}$ is set to 4 or 6 . An $a_{1}$-partition is then executed on $S_{n}$ to produce $n(n-1)$-vertices, and $F_{v}$ is partitioned accordingly. That is, two vertices in $F_{v}$ fall into the same subset if and only if they belong to the same ( $n$ 1)-vertex. The position $a_{2}$ is determined similarly. We simply let $a_{2}$ be a position where at least two vertices in some subset differ. Then an $a_{2}$-partition is executed on the $n(n-1)$-vertices to produce $n(n-1)(n-2)$-vertices, and every non-empty subset of $F_{v}$ is partitioned accordingly. The process is repeated until every non-empty subset contains one vertex, when the remaining positions are determined arbitrarily.

A path in a graph $G$ is said to be a hamiltonian path if it contains every vertex of $G$ exactly once [10]. In the following discussion, a path between two vertices, say $X$ and $Y$, are abbreviated to an $X-Y$ path.

Lemma 3. Suppose $n \geq 6$ and $\left|F_{v}\right| \leq n-3$. An $R_{4}$ with properties (P1), (P2), and (P3) can be generated in $S_{n}$.

Proof. Suppose $a_{1}, a_{2}, \ldots, a_{n-4}$ are a sequence of positions that satisfy Lemma 2 . We first construct an $R_{6}$ in $S_{n}$. When $n=6, S_{n}$ is an $R_{6}$ with only one 6-vertex. For $n>6$, an $R_{6}$ can be obtained by executing an $\left(a_{1}, a_{2}, \ldots, a_{n-6}\right)$ partition on $S_{n}$ as follows. Initially, an $a_{1}$-partition is applied to $S_{n}$, and so a $K_{n}^{n-1}$ results. An $R_{n-1}$ can be easily generated from the $K_{n}^{n-1}$. Then, for $j=2,3, \ldots, n-6$, an $R_{n}$ ${ }_{j}$ can be generated from an $R_{n-j+1}$ as explained below.

Suppose the $R_{n-j+1}=\left[A_{n-j+1,0}, A_{n-j+1,1}, \ldots, A_{n-j+1, n(n-1) \cdots(n-j+2)-}\right.$ 1]. Each $A_{n-j+1, k}$ forms a $K_{n-j+1}^{n-j}$ after an $a_{j}$-partition is executed on the $R_{n-j+1}$, where $0 \leq k \leq n(n-1) \cdots(n-j+2)-1$. Let $X_{k}$ and $Y_{k}$ denote a pair of distinct entry and exit $(n-j)$-vertices of $A_{n-j+1, k}$. It is easy to establish a hamiltonian $X_{k}-Y_{k}$ path in each $K_{n-j+1}^{n-j}$ formed by $A_{n-j+1, k}$. Then an $R_{n-j}$ can be generated if all the hamiltonian paths are interleaved with (n-j)-edges $\left(Y_{0}, X_{1}\right),\left(Y_{1}, X_{2}\right), \ldots,\left(Y_{n(n-1) \cdots(n-j+2)-1}, X_{0}\right)$. When $j=n-6$, an $R_{6}=\left[A_{6,0}, A_{6,1}, \ldots, A_{6, n(n-1) \cdots 7-1}\right]$ is obtained.

We continue to generate an $R_{5}$ from the $R_{6}$. When $n=6$, it is easy to generate an $R_{5}$ so that for arbitrary three consecutive 5-vertices $U=\left\langle u_{1} u_{2} \ldots u_{n}\right\rangle_{5}, V=\left\langle v_{1} v_{2} \ldots v_{n}\right\rangle_{5}$, and $W=\left\langle w_{1} w_{2} \ldots w_{n}\right\rangle_{5}$ in the $R_{5}, u_{\operatorname{dif}(U, V)} \neq w_{\operatorname{dif}(V, W)}$ holds. When $n>6$, each $A_{6, r}$ forms a $K_{6}^{5}$ after an $a_{n-5}$-partition is executed on the $R_{6}$, where $0 \leq r \leq n(n-1) \cdots 7-1$. Let $X_{r}$ and $Y_{r}$ denote a pair of distinct entry and exit 5-vertices of $A_{6, r}$. It is easy to establish a hamiltonian $X_{r}-Y_{r}$ path in the $K_{6}^{5}$ formed by $A_{6, r}$ whose first (last) two 5-vertices are connected to $A_{6,(r-1) \bmod n(n-1) \cdots 7}\left(A_{6,(r+1) \bmod n(n-1) \cdots 7}\right)$. All the hamiltonian paths interleaved with 5-edges $\left(Y_{0}, X_{1}\right),\left(Y_{1}\right.$, 
$\left.X_{2}\right), \ldots,\left(Y_{n(n-1) \cdots 7-1}, X_{0}\right)$ form an $R_{5}$. Besides, for arbitrary three consecutive 5-vertices $U=\left\langle u_{1} u_{2} \ldots u_{n}\right\rangle_{5}$, $V=\left\langle v_{1} v_{2} \ldots v_{n}\right\rangle_{5}$, and $W=\left\langle w_{1} w_{2} \ldots w_{n}\right\rangle_{5}$ in the $R_{5}, u_{\operatorname{dif}(U, V)} \neq$ $w_{\text {dif }(V, W)}$ holds. The reason is explained below.

Let $p=\operatorname{dif}(U, V)$ and $q=\operatorname{dif}(V, W)$. If $U=Y_{i}$ for some $0 \leq i \leq n(n-1)(n-2) \cdots 7-1$, then $V=X_{(i+1) \bmod n(n-1) \cdots 7}$ and $W$ is the second 5-vertex in the hamiltonian $X_{(i+1) \bmod n(n-1) \cdots 7^{-}} Y_{(i+1) \bmod }$ $n(n-1) \cdots 7$ path, which cause $p \neq a_{n-5}$ and $q=a_{n-5}$. Moreover, $W$ is connected to $A_{6, i}$ according to the construction above. Recall that the 5-vertex of $A_{6, i}$ that are not connected to $A_{6,(i+1) \bmod n(n-1) \ldots 7}$ is $\left\langle x_{1} \ldots x_{q-1} y_{p} x_{q+1} \ldots x_{n}\right\rangle_{5}$ and the 5-vertex of $A_{6,(i+1) \bmod n(n-1) \cdots 7}$ that are not connected to $A_{6, i}$ is $\left\langle y_{1} \ldots y_{q-}\right.$ ${ }_{1} x_{p} y_{q+1} \ldots y_{n}>{ }_{5}$, where $x_{q}=y_{p} \neq x_{p}=y_{q}$ and $x_{i}=y_{i}$ for all $1 \leq i \leq n$ and $i \notin\{p, q\}$. Hence, if $w_{q}=u_{p}$, then $W$ is not connected to $A_{6, i}$, which is a contradiction. If $W=X_{i}$ for some $0 \leq i \leq n(n-1)(n-$ 2) $\cdots 7-1$, then $u_{p} \neq w_{q}$ can be shown similarly. Otherwise, if $U, V$, and $W$ belong to the same 6-vertex, then $u_{p} \neq w_{q}$ because $p=q=a_{n-5}$.

We note that at most one 5 -vertex in the $R_{5}$ contains two vertex faults and the others each contain at most one vertex fault. If there are two 5 -vertices in the $R_{5}$ that each contain two or more vertex faults, then at most $n-5$ of the 5-vertices in the $R_{5}$ are faulty. This is a contradiction because the $R_{5}$, which results from executing an $\left(a_{1}, a_{2}, \ldots\right.$, $a_{n-5}$ )-partition on $S_{n}$, contains at least $n-4$ faulty 5 -vertices (see the paragraph after Lemma 2).

Next, we generate a desired $R_{4}$ from the $R_{5}$. Suppose the $R_{5}=\left[A_{5,0}, A_{5,1}, \ldots, A_{5, n(n-1) \cdots 6-1}\right]$. Without loss of generality, we assume that one 5-vertex, say $A_{5, k}(0 \leq k \leq n(n-$

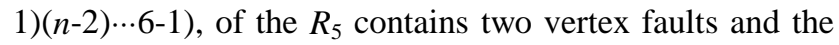
others each contain at most one vertex fault. An $a_{n-4^{-}}$ partition is executed on the $R_{5}$, and so each $A_{5, j}$ forms a $K_{5}^{4}$, where $0 \leq j \leq n(n-1) \cdots 6-1$. According to Lemma 1, each 4-vertex of $A_{5, k}$ is connected to $A_{5,(k-1) \bmod n(n-1) \cdots 6}$ or $A_{5,(k+1)}$ $\bmod n(n-1) \cdots 6$. We further assume, without loss of generality, that $A_{5, k}$ contains two faulty 4 -vertices, say $C$ and $E$. A hamiltonian path in the $K_{5}^{4}$ formed by $A_{5, k}$ can be established according to the following three cases.

Case 1. Both of $C$ and $E$ are connected to both $A_{5,(k-1) \bmod }$ $n(n-1) \cdots 6$ and $A_{5,(k+1) \bmod n(n-1) \cdots 6}$. Let $B(F)$ be the 4-vertex in $A_{5, k}$ that is not connected to $A_{5,(k+1) \bmod n(n-1) \cdots 6}\left(A_{5,(k-1) \bmod n(n-}\right.$ 1)‥6). A hamiltonian path is established as $(B, C, D, E, F)$, where $D$ is the other 4-vertex of $A_{5, k}$.

Case 2. One of $C$ and $E$ is connected to both $A_{5,(k-1) \bmod n(n-}$

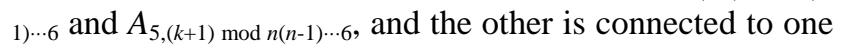
of $A_{5,(k-1) \bmod n(n-1) \cdots 6}$ and $A_{5,(k+1) \bmod n(n-1) \cdots 6}$. Without loss of generality, we assume $C$ is connected to both $A_{5,(k-1) \bmod n(n-}$ 1) $\cdots 6$ and $A_{5,(k+1) \bmod n(n-1) \cdots 6}$. If $E$ is connected to $A_{5,(k+1) \bmod n(n-}$ 1)‥6, a hamiltonian path is established as $(B, C, D, E, F)$, where $B$ is the 4-vertex of $A_{5, k}$ that is not connected to $A_{5}$, $(k+1) \bmod n(n-1) \cdots 6$, and $D$ and $F$ are the other two 4-vertices of $A_{5, k}$. If $E$ is connected to $A_{5,(k-1) \bmod n(n-1) \cdots 6}$, a hamiltonian path is established as $(D, E, F, C, B)$, where $B$ is the 4vertex of $A_{5, k}$ that is not connected to $A_{5,(k-1) \bmod n(n-1) \cdots 6}$, and $D$ and $F$ are the other two 4 -vertices of $A_{5, k}$.

Case 3. One of $C$ and $E$ is not connected to $A_{5,(k-1) \bmod n(n-1) \cdots 6}$, and the other is not connected to $A_{5,(k+1) \bmod n(n-1) \cdots 6}$ (it is impossible that $C$ and $E$ are not connected to $A_{5,(k-1) \bmod n(n-}$

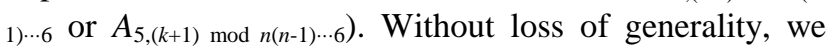
assume $C$ is not connected to $A_{5,(k+1) \bmod n(n-1) \cdots 6}$ and $E$ is not connected to $A_{5,(k-1) \bmod n(n-1) \cdots 6}$. A hamiltonian path is established as $(B, C, D, E, F)$, where $B, D$, and $F$ are the other three 4-vertices of $A_{5, k}$.

The first (last) 4-vertex in the hamiltonian paths above is the entry (exit) 4-vertex of $A_{5, k}$. Besides, the first (last) two 4-vertices are connected to $A_{5,(k-1) \bmod n(n-1) \cdots 6}\left(A_{5,(k+1) \bmod }\right.$ $n(n-1) \cdots 6$ ). Then we determine the entry and exit 4-vertices of $A_{5, j}$, denoted by $X_{5, j}$ and $Y_{5, j}$, respectively, for all $0 \leq j \leq n(n-$

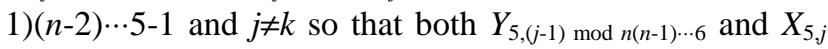
are not faulty and both $Y_{5, j}$ and $X_{5,(j+1) \bmod n(n-1) \cdots 6}$ are not faulty. It is not difficult to establish a hamiltonian $X_{5, j}-Y_{5, j}$ path in the $K_{5}^{4}$ formed by each $A_{5, j}$ whose first (last) two 4-vertices are connected to $A_{5,(j-1) \bmod n(n-1) \cdots 6}\left(A_{5,(j+1)} \bmod n(n-\right.$

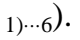

All the hamiltonian paths interleaved with 4-edges constitute an $R_{4}$. Clearly the $R_{4}$ has properties (P1) and (P3). Recall that for arbitrary three consecutive 5-vertices $U=\left\langle u_{1} u_{2} \ldots u_{n}\right\rangle_{5}, V=\left\langle v_{1} v_{2} \ldots v_{n}\right\rangle_{5}$, and $W=\left\langle w_{1} w_{2} \ldots w_{n}\right\rangle_{5}$ in the $R_{5}, u_{\operatorname{dif}(U, V)} \neq w_{\operatorname{dif}(V, W)}$ holds. With similar arguments, the $R_{4}$ has property (P2). This completes the proof. Q.E.D.

Lemma 4. Suppose $\left|F_{v}\right| \leq 1$. There is a healthy path of maximal length 4!-3 between arbitrary two adjacent healthy vertices of $S_{4}$.

Proof. Suppose $u$ and $v$ are arbitrary two adjacent healthy vertices of $S_{4}$, and let $f=f_{1} f_{2} f_{3} f_{4}$ denote the faulty vertex. Since the star graph is edge symmetric [2], we assume $u=u_{1} u_{2} u_{3} u_{4}=1234$ and $v=v_{1} v_{2} v_{3} v_{4}=3214$ for illustration. We have $f_{2} \neq u_{2}=v_{2}$ or $f_{4} \neq u_{4}=v_{4}$, for otherwise $f=u$ or $f=v$, which is a contradiction. We assume $f_{4} \neq u_{4}=v_{4}=4$, without loss of generality. After executing a 4-partition on $S_{4}, u$ and $v$ belong to $\langle * * * 4\rangle_{3}$ and $f$ belongs to $\langle * * * 1\rangle_{3}$ or $\langle * * * 2\rangle_{3}$ or $\langle * * * 3\rangle_{3}$.

If $f \in\langle * * * 1\rangle_{3}$, a healthy $u-v$ path of length 4 !-3 can be constructed as follows:

( $u=1234,2134,3124,1324,4321,3421,2431,4231$, $3241,1243,2143,4123,1423,2413,3412,1432$, 
$4132,3142,1342,4312,2314,3214=v)$ if $f=2341$;

$(u=1234,4231,3241,2341,4321,3421,1423,2413$, $4213,1243,2143,3142,1342,4312,3412,1432$, $4132,2134,3124,1324,2314,3214=v$ ) if $f=2431$;

$(u=1234,4231,2431,3421,4321,2341,1342,4312$, 3412 , 1432, 4132, 3142, 2143, 1243, 4213, 2413, $1423,4123,3124,1324,2314,3214=v)$ if $f=3241$;

$(u=1234,2134,3124,4123,1423,2413,4213,1243$, 2143, 3142, 1342, 4312, 3412, 1432, 2431, 4231, $3241,2341,4321,1324,2314,3214=v$ ) if $f=3421$;

$(u=1234,2134,4132,3142,1342,4312,3412,1432$, 2431, 3421, 4321, 2431, 3241, 1243, 4213, 2413, $1423,4123,3124,1324,2314,3214=v)$ if $f=4231$;

$(u=1234,2134,3124,4123,2143,1243,4213,2413$, 1423, 3421, 2431, 4231, 3241, 2341, 1342, 3142, $4132,1432,3412,4312,2314,3214=v)$ if $f=2341$.

If $f \in\langle * * * 2\rangle_{3}$ or $\langle * * * 3\rangle_{3}$, a healthy $u-v$ path of length 4 !-3 can be obtained similarly. Since the star graph is bipartite, the $u-v$ path is the longest in the worst case. Q.E.D.

We note that $S_{3}$ forms a ring of length six. The following two lemmas were shown in [32].

Lemma 5.[32] Suppose $U$ and $V$ are two adjacent 3vertices in $S_{n}$, and let $\left(c_{0}, c_{1}, \ldots, c_{5}\right)$ denote the ring formed by $U$. Then, the vertices of $U$ that are connected to $V$ are $c_{j}$ and $c_{(j+3) \text { mod } 6}$ for some $0 \leq j \leq 5$.

Lemma 6.[32] Suppose $U=\left\langle u_{1} u_{2} \ldots u_{n}\right\rangle_{3}$, $V=\left\langle v_{1} v_{2} \ldots v_{n}\right\rangle_{3}$, and $W=\left\langle w_{1} w_{2} \ldots w_{n}\right\rangle_{3}$ are three 3-vertices in $S_{n}$, and $V$ is adjacent to both $U$ and $W$. If $u_{\operatorname{dif}(U, V)} \neq w_{\operatorname{dif}(V, W)}$, then the two vertices of $V$ that are connected to $U$ are disjoint from the two vertices of $V$ that are connected to $W$.

Lemma 7. Suppose $n \geq 5$ and $\left|F_{v}\right| \leq n-3$. A healthy ring of maximal length $n !-2\left|F_{v}\right|$ can be generated from an $R_{4}$ in $S_{n}$ that owns properties (P1), (P2), and (P3).

Proof. Suppose an $R_{4}=\left[A_{0}, A_{1}, \ldots, A_{n(n-1) \cdots 5-1}\right]$ with properties (P1), (P2), and (P3) is obtained after executing an $\left(a_{1}, a_{2}, \ldots, a_{n-4}\right)$-partition on $S_{n}$. An $a_{n-3}$-partition is then executed on the $R_{4}$, and so each $A_{i}$ is partitioned into four 3 -vertices that form a $K_{4}^{3}$, where $a_{n-3} \in\{2,3, \ldots, n\}$ - $\left\{a_{1}\right.$, $\left.a_{2}, \ldots, a_{n-4}\right\}$ and $0 \leq i \leq n(n-1) \cdots 5-1$. According to Lemma 1 , each 3-vertex of $A_{i}$ is connected to $A_{(i-1) \bmod n(n-1) \cdots 5}$ or $A_{(i+1)}$ mod $n(n-1) \cdots 5$ because of property (P2). Since three 3 -vertices of $A_{i}$ are connected to $A_{(i-1) \bmod n(n-1) \cdots 5}$ and three 3-vertices of $A_{i}$ are connected to $A_{(i+1) \bmod n(n-1) \cdots 5}$, two 3-vertices of $A_{i}$ are connected to both $A_{(i-1) \bmod n(n-1) \cdots 5}$ and $A_{(i+1) \bmod n(n-1) \cdots 5}$.

We then determine the entry and exit 3-vertices of each $A_{i}$, denoted by $X_{i}$ and $Y_{i}$, respectively, as follows. For each faulty $A_{i}$, we let $X_{i}=Y_{i}=Q$, where $Q$ is a healthy 3vertex of $A_{i}$ that is connected to both $A_{(i-1) \bmod n(n-1) \cdots 5}$ and $A_{(i+1) \bmod n(n-1) \cdots 5}$. We note that $A_{i}$ contains one vertex fault (property $\mathrm{P}(1))$. Since $A_{(i-1) \bmod n(n-1) \cdots 5}$ and $A_{(i+1) \bmod n(n-1) \cdots 5}$ are healthy (property (P3)), $Y_{(i-1) \bmod n(n-1) \cdots 5}$ and $X_{(i+1) \bmod n(n-}$

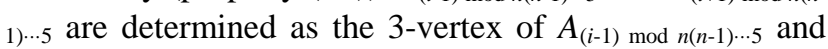
the 3-vertex of $A_{(i+1)} \bmod n(n-1) \cdots 5$, respectively, that are adjacent to $Q$. For every two consecutive healthy $A_{i}$ and $A_{(i+1) \bmod n(n-1) \cdots 5}, Y_{i}$ and $X_{(i+1) \bmod n(n-1) \cdots 5}$ are determined as two adjacent 3 -vertices that belong to $A_{i}$ and $A_{(i+1)} \bmod n(n-$ 1) $\cdots 5$, respectively.

All $X_{i}$ and $Y_{i}$ thus determined are healthy. Moreover, when $\quad X_{i}=Y_{i}, \quad u_{\operatorname{dif}\left(Y_{(i-1) \bmod n(n-1) \ldots 5}, X_{i}\right)} \neq$ $w_{\text {dif }\left(X_{i}, X_{(i+1) \bmod n(n-1) \cdots 5}\right)}$ holds, where $Y_{(i-1)} \bmod n(n-$ 1) $\cdots 5=\left\langle u_{1} u_{2} \ldots u_{n}\right\rangle_{4}$ and $X_{(i+1) \bmod n(n-1) \cdots 5}=\left\langle w_{1} w_{2} \ldots w_{n}\right\rangle_{4}$ are assumed. The reason is explained as follows. Suppose conversely

$$
u_{\text {dif }\left(Y_{(i-1) \bmod n(n-1) \cdots 5}, X_{i}\right)}=
$$

$w_{\operatorname{dif}\left(X_{i}, X_{(i+1) \bmod n(n-1) \cdots 5}\right)} \cdot \quad$ If $\quad \operatorname{dif}\left(Y_{(i-1)} \quad \bmod \quad n(n-1) \cdots 5\right.$, $\left.X_{i}\right)=\operatorname{dif}\left(X_{i}, X_{(i+1) \bmod n(n-1) \cdots 5}\right)$, then $Y_{(i-1) \bmod n(n-1) \cdots 5}=X_{(i+1) \bmod n}$ $(n-1) \cdots 5$, which is a contradiction. If $\operatorname{dif}\left(Y_{(i-1)} \bmod n(n-1) \cdots 5\right.$, $\left.X_{i}\right) \neq \operatorname{dif}\left(X_{i}, X_{(i+1) \bmod n(n-1) \cdots 5}\right)$, then there is a 3 -vertex of $A_{i}$ which is connected to neither of $A_{(i-1) \bmod n(n-1) \cdots 5}$ and $A_{(i+1)}$ mod $n(n-1) \cdots 5$. This is again a contradiction because each 3vertex of $A_{i}$ is connected to $A_{(i-1) \bmod n(n-1) \cdots 5}$ or $A_{(i+1) \bmod n(n-}$

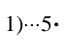

Next we generate a healthy ring of length $n !-2\left|F_{\nu}\right|$ by establishing healthy paths in $A_{0}, A_{1}, \ldots, A_{n(n-1) \cdots 5-1}$ in this sequence. First we construct a healthy path in $A_{0}$ as follows. Let $x_{0}$ be a healthy vertex of $X_{0}$ which is adjacent to a healthy vertex of $Y_{n(n-1) \cdots 5-1}$. When $X_{0}=Y_{0}$, $u_{\text {dif }\left(Y_{n(n-1) \cdots 5-1}, X_{0}\right)} \neq w_{\text {dif }\left(X_{0}, X_{1}\right)}$ holds for three consecutive 3-vertices $\quad Y_{n(n-1) \cdots 5-1}=\left\langle u_{1} u_{2} \ldots u_{n}\right\rangle_{4}, \quad X_{0}\left(=Y_{0}\right), \quad$ and $X_{1}=\left\langle w_{1} w_{2} \ldots w_{n}\right\rangle_{4}$. According to Lemmas 5 and 6 , there is a healthy vertex $y_{0}$ of $Y_{0}\left(=X_{0}\right)$ which is adjacent to both $x_{0}$ and $x_{1}$, where $x_{1}$ is a healthy vertex of $X_{1}$. If $A_{0}$ is healthy, it is not difficult to establish a healthy hamiltonian $x_{0}-y_{0}$ path in $A_{0}$. If $A_{0}$ is faulty, a healthy $x_{0}-y_{0}$ path of maximal length 4!-3 in $A_{0}$ can be established according to Lemma 4.

We then consider the situation of $X_{0} \neq Y_{0}$. Let $Q_{0}\left(L_{0}\right)$ be the 3-vertex of $A_{0}$ that is not connected to $A_{1}\left(A_{n(n-1) \cdots 5-1}\right)$, where $Q_{0} \neq Y_{0}\left(L_{0} \neq X_{0}\right)$. A healthy hamiltonian $X_{0}-Y_{0}$ path for the $K_{4}^{3}$ formed by $A_{0}$ is established as $\left(X_{0}, B, C, Y_{0}\right)$ if 
$Q_{0}=X_{0}$ and $L_{0}=Y_{0},\left(X_{0}, Q_{0}, C, Y_{0}\right)$ if $Q_{0} \neq X_{0}$ and $L_{0}=Y_{0},\left(X_{0}, B\right.$, $\left.L_{0}, Y_{0}\right)$ if $Q_{0}=X_{0}$ and $L_{0} \neq Y_{0}$, and $\left(X_{0}, Q_{0}, L_{0}, Y_{0}\right)$ if $Q_{0} \neq X_{0}$ and $L_{0} \neq Y_{0}$, where $B$ and $C$ are the other 3 -vertices of $A_{0}$.

Without loss of generality, we assume $\left(X_{0}, Q_{0}, L_{0}, Y_{0}\right)$ is the healthy hamiltonian $X_{0}-Y_{0}$ path. With the same arguments as the proof of Lemma 3, every three consecutive 3-vertices in $\left\{Y_{n(n-1) \cdots 5-1}, X_{0}, Q_{0}, L_{0}, Y_{0}, X_{1}\right\}$ satisfy property (P2). Since $A_{0}$ is healthy, according to Lemmas 5 and 6 there are four pairs of distinct healthy vertices, denoted by $\left\{x_{0}, p\right\},\{q, r\},\{s, t\}$, and $\left\{z, y_{0}\right\}$, of $X_{0}$, $Q_{0}, L_{0}$, and $Y_{0}$, respectively, so that every two consecutive vertices in $\left\{x_{0}, p, q, r, s, t, z, y_{0}, x_{1}\right\}$ are adjacent, where $x_{1}$ is a healthy vertex of $X_{1}$. Clearly, there are healthy hamiltonian $x_{0}-p, q-r, s-t$, and $z-y_{0}$ paths in $X_{0}, Q_{0}, L_{0}$, and $Y_{0}$, respectively. These healthy hamiltonian paths interleaved with edges $(p, q),(r, s)$, and $(t, z)$ constitute a healthy hamiltonian $x_{0}-y_{0}$ path in $A_{0}$.

Then we construct healthy paths in $A_{1}, A_{2}, \ldots, A_{n(n-1) \cdots 5-}$ 2 , sequentially. Suppose a healthy $x_{j-1}-y_{j-1}$ path in $A_{j-1}$ is obtained, where $1 \leq j \leq n(n-1) \cdots 5-2$. Very similar to the situation of $A_{0}$, a healthy path in $A_{j}$ can be constructed as follows. Let $x_{j}$ be the (healthy) vertex of $X_{j}$ that is adjacent to $y_{j-1}$. When $X_{j}=Y_{j}, u_{d i f\left(Y_{j-1}, X_{j}\right)} \neq w_{d i f\left(X_{j}, X_{j+1}\right)}$ holds for three consecutive 3-vertices $Y_{j-1}=\left\langle u_{1} u_{2} \ldots u_{n}\right\rangle_{4}, X_{j}\left(=Y_{j}\right)$, and $X_{j+1}=\left\langle w_{1} w_{2} \ldots w_{n}\right\rangle_{4}$. According to Lemmas 5 and 6 , there is a healthy vertex $y_{j}$ of $Y_{i}\left(=X_{j}\right)$ which is adjacent to both $x_{j}$ and a healthy vertex of $X_{j+1}$. If $A_{j}$ is healthy, there is a healthy hamiltonian $x_{j}-y_{j}$ path in $A_{j}$. If $A_{j}$ is faulty, a healthy $x_{j}-y_{j}$ path of maximal length 4 !-3 in $A_{j}$ can be established according to Lemma 4 . When $X_{j} \neq Y_{j}$, a hamiltonian $X_{j}-Y_{j}$ path for the $K_{4}^{3}$ formed by $A_{j}$ is first established. Then a healthy hamiltonian $x_{j}-y_{j}$ path in $A_{j}$ can be obtained by concatenating healthy hamiltonian paths for the four 3vertices of $A_{j}$, where $y_{j}$ is a healthy vertex of $Y_{j}$ and is adjacent to a healthy vertex of $X_{j+1}$.

Thus far we have established healthy paths in $A_{0}$, $A_{1}, \ldots, A_{n(n-1) \cdots 5-2}$, We continue to establish a healthy path in $A_{n(n-1) \cdots 5-1}$. Let $x_{n(n-1) \cdots 5-1}$ be the (healthy) vertex of $X_{n(n-1) \cdots 5-1}$ that is adjacent to $y_{n(n-1) \cdots 5-2}$, and $y_{n(n-1) \cdots 5-1}$ be the (healthy) vertex of $Y_{n(n-1) \cdots 5-1}$ that is adjacent to $x_{0}$. Without loss of generality, we use $\left(c_{0}, c_{1}, c_{2}, c_{3}, c_{4}, c_{5}\right)$ to represent $Y_{n(n-1) \cdots 5-}$ 1, where $c_{0}=y_{n(n-1) \cdots 5-1}$ is assumed. When $X_{n(n-1) \cdots 5-1}=Y_{n(n-1) \cdots 5-}$ $1, \quad u_{d i f\left(Y_{n(n-1) . .5-2}, X_{n(n-1) . .5-1}\right)} \neq w_{d i f\left(X_{n(n-1) . .5-1}, X_{0}\right)}$ holds for three consecutive 3-vertices $Y_{n(n-1) \cdots 5-2}=\left\langle u_{1} u_{2} \ldots u_{n}\right\rangle_{4}, X_{n(n-}$

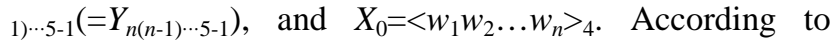
Lemmas 5 and $6, x_{n(n-1) \cdots 5-1}$ cannot be $c_{0}$ or $c_{3}$. Actually, $x_{n(n-}$ 1) $\cdots 5-1=c_{1}$ or $c_{5}$ (i.e., $x_{n(n-1) \cdots 5-1}$ and $y_{n(n-1) \cdots 5-1}$ are adjacent) as explained below.

If $x_{n(n-1) \cdots 5-1}=c_{2}$, a healthy path $\left(c_{2}, c_{1}, c_{0}\right)$ in $Y_{n(n-1) \cdots 5-1}$ together with all healthy paths that we have established in
$A_{0}, A_{1}, \ldots, A_{n(n-1) \cdots 5-2}$ form a ring of odd length in $S_{n}$. This is a contradiction because $S_{n}$ is a bipartite graph in which every ring has even length. Similarly, there is a contradiction for $x_{n(n-1) \cdots 5-1}=c_{4}$. Now that $x_{n(n-1) \cdots 5-1}$ and $y_{n(n-}$

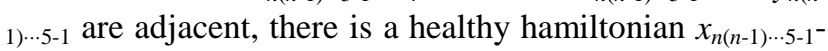
$y_{n(n-1) \cdots 5-1}$ path in $A_{n(n-1) \cdots 5-1}$ if $A_{n(n-1) \cdots 5-1}$ is healthy. If $A_{n(n-}$

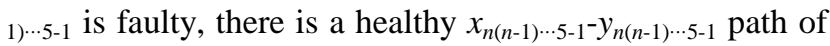
maximal length 4 !-3 in $A_{n(n-1) \cdots 5-1}$ according to Lemma 4.

When $X_{n(n-1) \cdots 5-1} \neq Y_{n(n-1) \cdots 5-1}$, a healthy hamiltonian $X_{n(n-}$

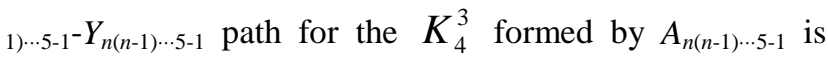
first established, similar to the situation of $A_{0}$. Without loss of generality, we assume $\left(X_{n(n-1) \cdots 5-1}, D, E, Y_{n(n-1) \cdots 5-1}\right)$ is the healthy hamiltonian $X_{n(n-1) \cdots 5-1}-Y_{n(n-1) \cdots 5-1}$ path. There are three pairs of distinct healthy vertices, denoted by $\left\{x_{n(n-}\right.$ 1) $\cdots 5-1, a\},\{b, c\}$, and $\{d, e\}$, in $X_{n(n-1) \cdots 5-1}, D$, and $E$, respectively, so that every two consecutive vertices in $\left\{x_{n(n-1) \cdots 5-1}, a, b, c, d, e, f\right\}$ are adjacent, where $f$ is a healthy vertex of $Y_{n(n-1) \cdots 5-1}$. Since $y_{n(n-1) \cdots 5-1}=c_{0}, f$ cannot be $c_{0}$ or $c_{3}$ according to Lemmas 5 and 6. Moreover, $f \in\left\{c_{2}, c_{4}\right\}$ will result in a ring of odd length in $S_{n}$, which is a contradiction. Hence, $f$ is adjacent to $y_{n(n-1) \cdots 5-1}$ (i.e., $f=c_{1}$ or $c_{5}$ ). It is easy to establish a healthy hamiltonian $x_{n(n-1)} \cdots 5-1-y_{n(n-1) \cdots 5-1}$ path in $A_{n(n-1) \cdots 5-1}$.

Clearly, all the healthy paths that we have established in $A_{0}, A_{1}, \ldots, A_{n(n-1) \cdots 5-1}$ together with edges $\left(y_{0}, x_{1}\right),\left(y_{1}\right.$, $\left.x_{2}\right), \ldots,\left(y_{n(n-1) \cdots 5-1}, x_{0}\right)$ constitute a healthy ring of length $n !-$ $2\left|F_{v}\right|$. Since $S_{n}$ is bipartite with two partite sets of equal size, the ring is the longest in the worst case. Q.E.D.

Theorem 1. Suppose $n \geq 4$ and $\left|F_{\nu}\right| \leq n-3 . S_{n}$ can embed a healthy ring of maximal length $n !-2\left|F_{v}\right|$.

Proof. We show that $S_{n}$ contains a healthy ring of maximal length $n !-2\left|F_{v}\right|$ according to different values of $n$. When $n=4,\left|F_{v}\right| \leq 1$. By the aid of Lemma 4 , there is a healthy ring of maximal length $4 !-2\left|F_{v}\right|$ in $S_{4}$.

When $n=5,\left|F_{v}\right| \leq 2$. Without loss of generality, we assume $\left|F_{v}\right|=2$. According to Lemma 2, the two vertex faults of $S_{5}$ will be distributed to two different 4-vertices after an $a_{1}$-partition is executed, where $2 \leq a_{1} \leq 5$. Then an $R_{4}$ is established so that the two faulty 4-vertices are not adjacent in the $R_{4}$. Clearly the $R_{4}$ satisfies properties (P1) and (P3). Besides, it is not difficult to check that the $R_{4}$ also satisfies property (P2). By Lemma 7, a healthy ring of maximal length $5 !-2\left|F_{v}\right|$ can be generated from the $R_{4}$.

When $n \geq 6$, an $R_{4}$ with properties (P1), (P2), and (P3) is first obtained by Lemma 3 . Then, a healthy ring of maximal length $n !-2\left|F_{v}\right|$ can be generated by Lemma 7 . Q.E.D.

\section{Concluding remarks}


In this paper, we have shown that a healthy ring of length $n !-2\left|F_{v}\right|$ can be embedded onto an $n$-dimensional star graph with $\left|F_{v}\right| \leq n-3$ vertex faults. This improves Tseng et al.'s result by lengthening the embedded ring from $n !-4\left|F_{v}\right|$ to $n !-2\left|F_{v}\right|$. Since the star graph is regular of degree $n-1$ and is bipartite with two partite sets of equal size, our result achieves optimality in the worst case.

In [32], Tseng et al. also showed that an $n$ dimensional star graph with $\left|F_{v}\right|$ vertex faults and $\left|F_{e}\right|$ edge faults can embed a healthy ring of length at least $n !-4\left|F_{v}\right|$ if $\left|F_{v}\right|+\left|F_{e}\right| \leq n-3$. With our result, the embedded ring can be lengthened from $n !-4\left|F_{v}\right|$ to $n !-2\left|F_{v}\right|$ as well.

\section{References}

[1] S. B. Akers, D. Harel, and B. Krishnamurthy, "The star graph: an attractive alternative to the $n$-cube," Proceedings of the International Conference on Parallel Processing, 1986, pp. 216-223.

[2] S. B. Akers, B. Krishnamurthy, "A Group-theoretic model for symmetric interconnection networks," IEEE Transactions on Computers, vol. 38, no. 4, pp. 555-566, 1989.

[3] N. Bagherzadeh, M. Dowd, and N. Nassif, "Embedding an arbitrary tree into the star graph," IEEE Transactions on Computers, vol. 45, no. 4, pp. 475-481, 1996.

[4] J. C. Bermond, Ed., Interconnection Networks, a special issue of Discrete Applied Mathematics, vol. 37+38, 1992.

[5] P. Berthome, A. Ferreira, and S. Perennes, "Optimal information dissemination in star and pancake networks," IEEE Transaction on Parallel and Distributed Systems, vol. 7, no. 12, pp. 12921300, 1996.

[6] J. Bruck, R. Cypher, and C. T. Ho, "Fault-tolerant meshes and hypercubes with minimal numbers of spares," IEEE Transaction on Computers, vol. 42, no. 9, pp. 1089-1104, 1993.

[7] J. Bruck, R. Cypher, and C. T. Ho, "Fault-tolerant de Bruijn and shuffle-exchange networks," IEEE Transaction on Parallel and Distributed Systems, vol. 5, no. 5, pp. 548-553, 1994.

[8] J. Bruck, R. Cypher, and C. T. Ho, "On the construction of fault-tolerant cube-connected cycles networks," Journal of Parallel and Distributed Computing, vol. 25, pp. 98-106, 1995

[9] J. Bruck, R. Cypher, and C. T. Ho, "Wildcard dimensions, coding theory and fault-tolerant meshes and hypercubes," IEEE Transaction on Computers, vol. 44, no. 1, pp. 150-155, 1995.

[10] F. Buckley and F. Harary, Distance in Graphs, Addition-Wesley, 1989.
[11] M. Y. Chan, F. Y. L. Chin, and C. K. Poon, "Optimal simulation of full binary trees on faulty hypercubes," IEEE Transaction on Parallel and Distributed Systems, vol. 6, no. 3, pp. 269-286, 1995.

[12] K. Day and A. Tripathi, "A comparative study of topological properties of hypercubes and star graphs," IEEE Transactions on Parallel and Distributed Systems, vol. 5, no. 1, pp. 31-38, 1994.

[13] K. Diks and A. Pele, "Efficient gossiping by packets in networks with random faults," SIAM Journal on Discrete Mathematics, vol. 9, no. 1, pp. 7-18, 1996.

[14] A. H. Esfahanian and S. L. Hakimi, "Fault-tolerant routing in de Bruijn communication networks," IEEE Transactions on Computers, vol. C-34, no. 9, pp. 777-788, 1985.

[15] P. Fragopoulou and S. G. Akl, "A parallel algorithm for computing Fourier transforms on the star graph," IEEE Transactions on Parallel and Distributed Systems, vol. 5, no. 5, pp. 525-531, 1994.

[16] P. Fragopoulou and S. G. Akl, "Optimal communication algorithms on star graphs using spanning tree constructions," Journal of Parallel and Distributed Computing, vol. 24, pp. 55-71, 1995.

[17] D. F. Hsu, Interconnection Networks and Algorithms, a special issue of Networks, vol. 23, no. 4, 1993.

[18] J. S. Jwo, S. Lakshmivarahan, and S. K. Dhall, "Embedding of cycles and grids in star graphs," Journal of Circuits, Systems, and Computers, vol. 1, no. 1, pp. 43-74, 1991.

[19] H. K. Ku and J. P. Hayes, "Optimally edge faulttolerant trees," Networks, vol. 27, pp. 203-214, 1996.

[20] S. Latifi and N. Bagherzadeh, "Hamiltonicity of the Clustered-star graph with embedding applications," Proceedings of the International Conference on Parallel and Distributed Processing Techniques and Applications, 1996, pp. 734-744.

[21] A. C. Liang, S. Bhattacharya, and W. T. Tsai, "Fault-tolerant multicasting on hypercubes," Journal of Parallel and Distributed Computing, vol. 23, pp. 418-428, 1994.

[22] A. Mann and A. K. Somani, "An efficient sorting algorithm for the star graph interconnection network," Proceedings of the International Conference on Parallel Processing, vol. III, 1990, pp. 1-8.

[23] Z. Miller, D. Pritikin, and I. H. Sudborough, "Near embeddings of hypercubes into Caley graphs on the 
symmetric group," IEEE Transactions on Computers, vol. 43, no. 1, pp. 13-22, 1994.

[24] K. Qiu and S. G. Akl, "Load balancing and selection on the star and pancake interconnection networks," Proceedings of the $26^{\text {th }}$ Annual Hawaii International Conference on System Sciences, 1993, pp. 235-242.

[25] K. Qiu, S. G. Akl, and H. Meijer, "On some properties and algorithms for the star and pancake interconnection networks," Journal of Parallel and Distributed Computing, vol. 12, pp. 16-25, 1994.

[26] K. Qiu, S. G. Akl, and I. Stojmenovic, "Data communication and computational geometry on the star and pancake networks," Proceedings of the IEEE Symposium on Parallel and Distributed Processing, 1991, pp. 415-422.

[27] S. Ranka, J. C. Wang, and N. Yeh, "Embedding meshes on the star graph," Journal of Parallel and Distributed Computing, vol. 19, pp. 131-135, 1993.

[28] Y. Rouskov, S. Latifi, and P. K. Srimani, "Conditional fault diameter of star graph networks," Journal of Parallel and Distributed Computing, vol. 33, pp. 91-97, 1996.

[29] R. A. Rowley and B. Bose, "Distributed ring embedding in faulty De Bruijn networks," IEEE Transaction on Computers, vol. 46, no. 2, pp. 187190, 1997.

[30] D. K. Saikia and R. K. Sen, "Two ranking schemes for efficient computation on the star interconnection network," IEEE Transactions on Parallel and Distributed Systems, vol. 7, no. 4, pp. 321-327, 1996.

[31] J. P. Sheu, C. T. Wu and T. S. Chen, "An optimal broadcasting algorithm without message redundancy in star graphs," IEEE Transactions on Parallel and Distributed Systems, vol. 6, no. 6, pp. 653-658, 1995.

[32] Y. C. Tseng, S. H. Chang, and J. P. Sheu, "Faulttolerant ring embedding in star graphs," IEEE Transactions on Parallel and Distributed Systems, to appear.

[33] J. Wu, "Safety levels-an efficient mechanism for achieving reliable broadcasting in hypercubes," IEEE Transaction on Computers, vol. 44, no. 5, pp. 702-706, 1995.

[34] J. Wu, "Reliable unicasting in faulty hypercubes using safety levels," IEEE Transaction on Computers, vol. 46, no. 2, pp. 241-247, 1997.

[35] P. J. Yang, S. B. Tien, and C. S. Raghavendra, "Embedding of rings and meshes onto faulty hypercubes using free dimensions," IEEE Transaction on Computers, vol. C-43, no. 5, pp. 608-613, 1994. 